\section{Jejunoduodenal Fistula due to Penetrating Peptic Ulcer after Roux-en-Y Hepaticojejunostomy}

Biliary-enteric communications between the gallbladder and duodenum, or between the common bile duct and the duodenum, are infrequently reported. They are mainly caused by perforation of gallstones into an otherwise normal duodenum, or by peptic ulceration from the duodenum into the biliary tree, respectively [1,2]. Iatrogenic fistulas resulting from the passage of bougies or dilators into the duodenum during exploration of the common bile duct have also been mentioned in the literature [3]. A 55year-old man underwent hilar liver resection with left hepatectomy and Roux-en-Y hepaticojejunostomy for a Klatskin cholangiocarcinoma. Intraoperatively, the duodenum appeared normal. Uncommon postoperative epigastric symptoms led to a gastroduodenal endoscopy examination and diagnosis of a duodenal ulcer. The ulcer was attributed to Helicobacter pylori infection. Eradication therapy was carried out following the Italian schema. The patient was finally discharged on postoperative day 15 . Two months later, the patient returned, with epigastric pain and signs of cholangitis. A gastroduodenal endoscopy examination raised a suspicion of a jejunoduodenal fistula between the proximal duodenum and the Roux limb, probably caused by penetration of the peptic ulcer (Figures 1,2). The patient received therapy with ciprofloxacin, ursodeoxycholic acid, and omeprazole. A 6-week period of endoscopic observation showed that the peptic lesion was healing. However, the

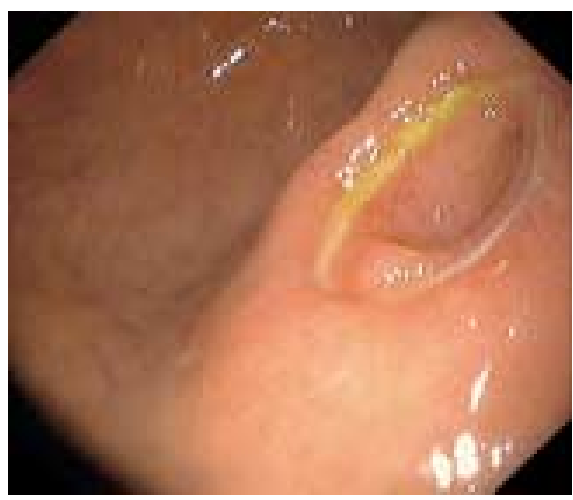

Figure 1 Endoscopic image raising a suspicion of fistula. fistula persisted, with the patient having symptoms of relapsing ascending cholangitis. The duodenal lesion and fistula were therefore finally excised, and direct closure of the fistula opening on the duodenal wall was carried out. At the time of writing, a year after the last operation, the patient is living normally and is free of symptoms. In view of the numbers of Roux-en-Y reconstructions carried out in digestive tract surgery worldwide [4], the masked clinical presentation of the fistula in the present case should be noted in order to facilitate the differential diagnosis in cases of unexplained postoperative epigastric pain associated with cholangitis. Despite an extensive literature search, no
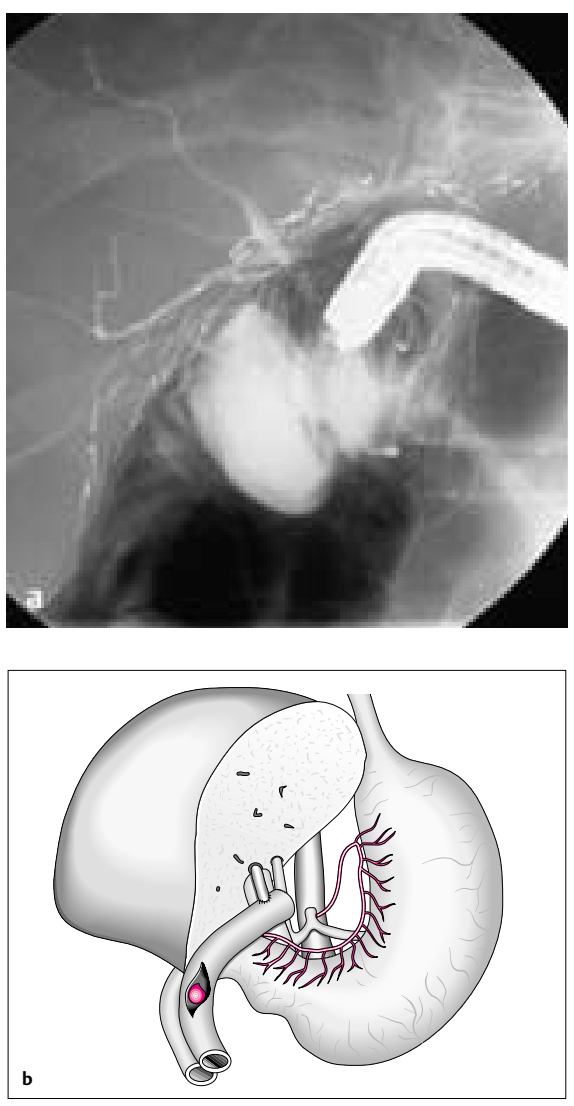

Figure 2 a Radiological confirmation of the fistula between the proximal duodenum and the Roux limb. The diagnosis was made by administering contrast through an endoscopic retrograde cholangiography catheter; an upper gastrointestinal barium study did not demonstrate the fistula. b Schematic demonstration of the fistula. previous reports of a jejunoduodenal fistula after Roux-en-Y reconstruction have been identified.

\section{Acknowledgement}

Dr. Sotiropoulos was supported by the Alexander Onassis Public Benefit Foundation.

\section{G. C. Sotiropoulos ${ }^{1}$, K. J. Oldhafer ${ }^{1}$,}

N. R. Frühauf ${ }^{1}$, U. Treichel ${ }^{2}$,

U. A. Clauer ${ }^{1}$, E. I. Brokalaki ${ }^{1}$,

\section{E. Broelsch ${ }^{1}$}

${ }^{1}$ Dept. of General Surgery

and Transplantation

${ }^{2}$ Dept. of Gastroenterology and Hepatology, Essen University Hospital, Essen,

Germany

\section{References}

${ }^{1}$ Feller ER, Warshaw AL, Schapiro RH. Observations on management of choledochoduodenal fistula due to penetrating peptic ulcer. Gastroenterology 1980; 78: 126 - 131

${ }^{2}$ Iso Y, Yoh R, Okita K et al. Choledochoduodenal fistula: a complication of a penetrated duodenal ulcer. Hepatogastroenterology 1996; 43: 489-491

${ }^{3}$ Hunt DR, Blumgart LH. Iatrogenic choledochoduodenal fistula: an unsuspected cause of post-cholecystectomy symptoms. Br J Surg 1980; 67: 10-13

${ }^{4}$ Besson A. The Roux-Y loop in modern digestive tract surgery. Am J Surg 1985; 149: $656-664$

\section{Corresponding Author}

\section{G. C. Sotiropoulos, M.D.}

Dept. of General Surgery and Transplantation

Essen University Hospital

Hufelandstrasse 55

45122 Essen

Germany

Fax: $\quad$ +49-201-723-1113

E-mail: georgios.sotiropoulos@ med.uni-essen.de 